\title{
Reflets
}

Revue ontaroise d'intervention sociale et communautaire

\section{Les éléments clés de la supervision avec Louise Villeneuve : vidéo produite et réalisée par Pat Tobin, coordonnatrice des stages et admissions, École de service social, Université Laurentienne}

\section{Jean-Marc Bélanger}

Volume 6, numéro 1, printemps 2000

Approches d'intervention : définir et renouveler nos pratiques

URI : https://id.erudit.org/iderudit/026311ar

DOI : https://doi.org/10.7202/026311ar

Aller au sommaire du numéro

Éditeur(s)

Reflets : Revue ontaroise d'intervention sociale et communautaire

ISSN

1203-4576 (imprimé)

1712-8498 (numérique)

Découvrir la revue

Citer ce compte rendu

Bélanger, J.-M. (2000). Compte rendu de [Les éléments clés de la supervision avec Louise Villeneuve : vidéo produite et réalisée par Pat Tobin, coordonnatrice des stages et admissions, École de service social, Université Laurentienne]. Reflets, 6(1), 291-294. https://doi.org/10.7202/026311ar

Tous droits réservés (C) Reflets : Revue ontaroise d'intervention sociale et communautaire, 2000
Ce document est protégé par la loi sur le droit d'auteur. L'utilisation des services d'Érudit (y compris la reproduction) est assujettie à sa politique d'utilisation que vous pouvez consulter en ligne. 


\section{Les éléments clés de la supervision avec Louise Villeneuve}

Vidéo produite et réalisée par Pat Tobin, coordonnatrice des stages et admissions,

École de service social, Université Laurentienne

par Jean-Marc Bélanger,

professeur agrégé, École de service social, Université Laurentienne

Dans cette vidéo, Louise Villeneuve offre les éléments clés de la supervision de stage, soit cette période de formation pratique qui s'insère dans les programmes de service social et qui s'effectue dans un milieu de travail. Il s'agit d'une expérience d'apprentissage supervisé qui vise l'acquisition de connaissances, d'habiletés et d'attitudes nécessaires à l'exercice de la profession de travailleuses et de travailleurs sociaux. Cette vidéo a été filmée lors d'un atelier offert par LouiseVilleneuve à des superviseures et des superviseurs de stage à l'École de service social de l'Université Laurentienne. Elle comporte trois parties.

Dans la vidéo, Louise Villeneuve aborde certains éléments de la supervision. D'abord, elle présente certaines distinctions terminologiques. Ensuite, elle tente de définir le rôle de la superviseure ou du superviseur et, finalement, elle aborde les éléments de la préparation pour les rencontres de supervision.

Ainsi, dans la première partie, Villeneuve insiste sur les trois fonctions que doit remplir toute supervision: la fonction d'enseignement (transmission des savoirs), la fonction de soutien (appui à la ou au stagiaire) et la fonction administrative (gestion). Elle distingue ensuite au moyen d'exemples concrets les notions de supervision, consultation, formation et psychothérapie. Pour ce faire, elle utilise les expériences des superviseures et des 
superviseurs qui participent à l'atelier pour faire le pont entre la théorie et la pratique. Cette approche valorise les personnes et leur permet de prendre conscience de l'importance du rôle des superviseures et des superviseurs. Cela dit, suivonsVilleneuve dans l'établissement de ces distinctions. Elle présente d'abord la supervision comme un processus qui permet une analyse réflexive autour d'un objet de travail, où les stagiaires mettent de côté les jugements (le pourquoi) afin de favoriser la découverte de soi (le comment). PourVilleneuve, la supervision vise le développement de compétences chez les stagiaires. Elle insiste sur l'importance de rencontres périodiques pour effectuer une supervision ordonnée et structurée qui permet le développement des compétences et de la relation entre superviseure et supervisée. Ainsi, le caractère continu de la supervision la distingue de la consultation qui est, à ses yeux, un processus plutôt ponctuel visant à répondre ou à donner un conseil, un avis dans le but de faciliter la prise de décision. Quant à la formation, elle l'associe à un ensemble d'activités d'ordre scolaire. À cet égard, la supervision des stagiaires se situe en aval de cette formation, venant la compléter à l'occasion, mais surtout en établissant le passage entre la théorie et la pratique. Pour Villeneuve, la supervision ne doit pas reprendre la formation de base déjà reçue en classe, mais certains éléments peuvent être revus lors de la supervision et selon les besoins des étudiantes et des étudiants. En dernier lieu, la psychothérapie ou la relation d'aide est le processus par lequel le travailleur et la travailleuse sociale peuvent traiter des conflits personnels, ce qui suppose une maitrise certaine des compétences.

La deuxième partie de la vidéo traite du rôle et des responsabilités de la personne qui supervise le stage. Dans l'ensemble, cette personne est responsable du développement professionnel des stagiaires. À titre d'exemple, il importe que les personnes qui supervisent ne prennent pas pour acquis certains éléments de base si bien intériorisés qu'ils sont devenus une seconde nature. Par ailleurs, elles et ils se doivent d'expliquer les politiques de l'agence ou de l'organisme et de donner des explications sur sa culture organisationnelle. En ce sens, il faut que les superviseures et les superviseurs veillent à ce que les 
stagiaires comprennent le contexte et le climat dans lequel elles ou ils sont appelés à travailler. De plus, les superviseures et les superviseurs sont responsables d'assigner et de prévoir les tâches des stagiaires. Cela permettra aux stagiaires d'atteindre un rythme de travail naturel sans pour autant s'attendre que les stagiaires deviennent des professionnels de l'agence. Il faut le rappeler : ne pas savoir et l'erreur font partie de tout apprentissage. Beaucoup d'autres responsabilités incombent aux superviseures et superviseurs et elles sont illustrées dans la vidéo.

Le partenariat entre la superviseure ou le superviseur de stage et la consultante ou le consultant académique est important. En effet, selon Villeneuve, la personne qui supervise n'a pas toujours toute l'expérience nécessaire du processus de supervision et elle ne doit pas hésiter à faire appel à la consultante ou au consultant. Cette personne ressource joue un rôle pédagogique dans l'encadrement du stage. Par exemple, elle aide souvent les stagiaires à élaborer des objectifs d'apprentissage précis et à déterminer les tâches correspondantes. Ces éléments peuvent éventuellement faire l'objet de contrats d'apprentissage et être évalués. Si des difficultés devaient survenir lors du stage, la responsabilité d'intervenir reviendrait à la consultante ou au consultant afin de s'assurer des apprentissages.

En bref, le rôle de supervision comprend trois éléments : être en mesure de créer un climat d'échange dans l'apprentissage, savoir communiquer les savoirs acquis et pouvoir appuyer la ou le stagiaire dans ses nouveaux apprentissages.

La troisième partie de la bande vidéo illustre comment se préparer pour la supervision. Ici, Villeneuve invite l'auditoire à participer à un exercice qui permet de discuter des questions sur le vif. Quatre éléments ressortent de la discussion pour la supervision. Il importe de réserver du temps pour faire la supervision, de prévoir la durée et la fréquence de la supervision, de planifier la mise à jour du contrat d'apprentissage, du journal de bord du stagiaire et de prendre le temps d'accueillir les commentaires des stagiaires dans une relation qui se veut réciproque. Dans la vidéo, en plus de discuter des tâches de la superviseure et du superviseur, la discussion porte aussi sur la 
préparation que les stagiaires doivent faire pour permettre des rencontres de supervision efficaces et fructueuses.

Voilà quelques-uns des éléments qu'on retrouve dans cette vidéo sur la supervision de stage. Et la vidéo est construite de façon pratique pour la personne qui supervise les stages puisqu'il est possible de visionner la partie désirée en fonction de ses besoins. En plus de contenir du matériel à jour sur la supervision, la vidéo permet de repérer facilement un point ou une dimension particulière. Finalement, Villeneuve se sert d'exemples concrets pour illustrer des techniques efficaces de supervision.

En somme, cette vidéo est un outil pédagogique pratique pour la supervision. Il peut aussi servir de matériel complémentaire à certains cours, tant au niveau des études supérieures qu'au niveau du baccalauréat. Les principes de base présentés sont bien expliqués et bien illustrés à l'aide d'une série d'exemples concrets. Ces principes peuvent enrichir d'autres professions dans le domaine de l'intervention sociale. C'est un outil indispensable à quiconque fait ou veut faire de la supervision de stages pratiques dans le cadre de son domaine académique ou de sa profession.

La professeure Tobin, qui a produit et réalisé cette bande vidéo, a fait un excellent travail et elle a réussi à capter sur pellicule, en 75 minutes, les éléments de base d'une bonne supervision s'adressant autant aux novices qu'aux plus aguerris d'entre nous. C'est pourquoi nous n'avons aucune hésitation à recommander l'utilisation de cette vidéo comme outil pédagogique de premier plan pour le domaine de la supervision ${ }^{1}$.

\section{Note}

1. On peut se procurer la bande vidéo à l'adresse suivante : École de service social, Université Laurentienne, Chemin du lac Ramsey, Sudbury, Ontario, P3E 2C6. On peut aussi communiquer par téléphone au (705) 675-1151 poste 5050, par télécopieur au (705) 671-3832 ou par courriel àmpharand@nickel.laurentian.ca 